\title{
Quantum Mechanics of Complex Octic Potential in One Dimension
}

\author{
Ram Mehar Singh \\ Department of Physics, Chaudhary Devi Lal University, Sirsa, India \\ Email: dixit_rammehar@yahoo.co.in
}

Received November 11, 2012; revised December 19, 2012; accepted December 29, 2012

\begin{abstract}
For gaining further insight into the nature of the eigenspectra of a complex octic potential [say $V(x)=\sum_{i=0}^{8} a_{i} x^{i}$ ], we investigate the quasi exact solutions of the Schrödinger equation in an extended complex phase space characterized by $x=x_{1}+i p_{2}, p=p_{1}+i x_{2}$. The analyticity property of the eigenfunction alone is found sufficient to throw light on the nature of eigenvalues and eigenfunction of a system. Explicit expressions of eigenvalues and eigenfunctions for the ground state as well as for the first excited state of a complex octic potential and its variant are worked out. It is found that imaginary part of the eigenvalue $\left(E_{i}\right)$ turns out to be zero for real coupling parameters, whereas it becomes non-zero for complex coupling parameters. However, the $P T$-symmetric version of a non-hermitian Hamiltonian possesses the real eigenvalue even if coupling parameters in the potential are complex.
\end{abstract}

Keywords: Schrödinger Equation; Complex Potential; PT-Symmetry; Ansatz

\section{Introduction}

Though complex potentials are in practice for a long time, the quantum mechanics of such potentials has not been studied to a desired level. It is only in the last few years that study of complex potentials has become important for better theoretical understanding of some newly discovered phenomena in Physics and Chemistry like phenomena pertaining to resonance scattering in atomic, molecular, and nuclear Physics and to some chemical reactions [1,2]. Besides some general studies of a complex Hamiltonian in nonlinear domain [3-5], efforts have been made to study both classical as well as quantum aspects [6-8] of the one-dimensional complex Hamiltonian systems. In the classical context, $H(x, p)$ becomes the function of two complex variables and analyticity property of the Hamiltonian leading to a class of integrable systems. In the quantum context, the analyticity of $H(x, p)$ is translated into the complex potential $V(x)$. It is observed that complex Hamiltonian is no longer hermitian and ordinarily does not guarantee for real eigenvalues; however, in its $P T$-symmetric form [9-11], the system is found to exhibit real eigenvalue spectrum [12]. The reality of the spectrum is a direct consequence of the combined action of the parity and time reversal invariance of Hamiltonian [13]. The parity operator $\hat{P}$ and the time reversal operator $\hat{T}$ are defined by the action of position and momentum operator as
$\hat{P}: \hat{x} \rightarrow-\hat{x} ; \hat{p} \rightarrow-\hat{p} \quad$ and $\quad \hat{T}: \hat{x} \rightarrow \hat{x} ; \hat{p} \rightarrow-\hat{p} ; \hat{i} \rightarrow-\hat{i}$. The non-hermitian $P T$-symmetric Hamiltonians play an important role in various fields like superconductivity, population biology, quantum cosmology, condensed matter Physics and quantum field theory etc $[5,8]$.

In literature, the complexity of the Hamiltonian is introduced in several ways but here we use the scheme given by Xavier and de Aguir [10], which is used to develop an algorithm for the computation of semi classical coherent state propagator, to transform potentials using extended complex phase space approach (ECPSA). In this approach, the transformations of the positions and the momenta variables are defined as

$$
x=x_{1}+i p_{2}, p=p_{1}+i x_{2} .
$$

Here, the variables $\left(x_{1}, p_{1}, x_{2}, p_{2}\right)$ are considered as some sort of coordinate-momentum interactions for a dynamical system. For the dimensional considerations, there should be a constant "d" in (1) i.e. $x=x_{1}+i d p_{2}$, $p=p_{1}+i d^{-1} x_{2}$, etc and for simplicity, we select $d=1$. It is to be noted that in this complexifying scheme, the degrees of freedom of the underlying system just become double. Recently, some efforts have been made to solve the Schrödinger equation (SE) for low order polynomial potentials in real as well as complex phase space [13-15]; however, no attempt has been made for higher order potentials. With the same spirit and to 
expand the domain of applications, we find the quasiexact solutions of the SE for a coupled complex octic potential and its variant.

The paper is organized as follows: in Section 2, we carry out the mathematical formulation of the ECPSA to compute eigenvalue spectra of the SE in one dimension. Under such mathematical prescription, the ground state solutions are described in Section 3, whereas excited state solutions are addressed in Section 4. Finally, concluding remarks are presented in Section 5.

\section{The Methods}

For, one-dimensional complex Hamiltonian system $H(x, p)$, the ASE $(=m=1)$ is given by

$$
\hat{H}(x, p) \psi(x)=E \psi(x),
$$

where

$$
\hat{H}(x, p)=-\frac{1}{2} \frac{\mathrm{d}^{2}}{\mathrm{~d} x^{2}}+V(x) .
$$

After employing transformation (1), one gets

$$
\frac{\mathrm{d}}{\mathrm{d} x}=\frac{1}{2}\left(\frac{\partial}{\partial x_{1}}-i \frac{\partial}{\partial p_{2}}\right), \frac{d}{d p}=\frac{1}{2}\left(\frac{\partial}{\partial p_{1}}-i \frac{\partial}{\partial x_{2}}\right) .
$$

Under the transformation (1), momentum operator becomes : $p=p_{1}+i x_{2}=\frac{-i}{2}\left(\frac{\partial}{\partial x_{1}}-i \frac{\partial}{\partial p_{2}}\right)$, where $p_{1}=\frac{-1}{2} \frac{\partial}{\partial p_{1}}, x_{2}=-\frac{\partial}{\partial x_{1}}$.

The real and imaginary parts of $V(x), \psi(x)$ and $E$ are written as

$$
\begin{gathered}
V(x)=V_{r}\left(x_{1}, p_{2}\right)+i V_{\mathrm{i}}\left(x_{1}, p_{2}\right), \\
\psi(x)=\psi_{r}\left(x_{1}, p_{2}\right)+i \psi_{\mathrm{i}}\left(x_{1}, p_{2}\right), \\
E=E_{r}+i E_{\mathrm{i}},
\end{gathered}
$$

where the subscript " $r$ " and " $i$ " denotes the real and imaginary parts of the corresponding quantities and other subscripts to these quantities separated by comma denotes the partial derivatives of the quantity concerned. On employing Equations (1), (3) and (5a)-(5e) in Equation (2), then after separating real and imaginary parts in the final result, the following pair of partial differential equations is obtained

$$
\begin{aligned}
& -\frac{1}{8}\left(\psi_{r, x_{1} x_{1}}-\psi_{r, p_{2} p_{2}}+2 \psi_{\mathrm{i}, x_{1} p_{2}}\right) \\
& +V_{r} \psi_{r}-V_{\mathrm{i}} \psi_{\mathrm{i}}=E_{r} \psi_{r}-E_{\mathrm{i}} \psi_{\mathrm{i}}, \\
& -\frac{1}{8}\left(\psi_{\mathrm{i}, x_{1} x_{1}}-\psi_{\mathrm{i}, p_{2} p_{2}}+2 \psi_{r, x_{1} p_{2}}\right) \\
& +V_{r} \psi_{\mathrm{i}}+V_{\mathrm{i}} \psi_{r}=E_{r} \psi_{\mathrm{i}}+E_{\mathrm{i}} \psi_{r} .
\end{aligned}
$$

Under Cauchy-Riemann condition, the analyticity property of the wavefunction $\psi(x)$ implies

$$
\psi_{r, x_{1}}=\psi_{\mathrm{i}, p_{2}} ; \psi_{r, p_{2}}=-\psi_{\mathrm{i}, x_{1}} .
$$

By imposing the analyticity condition (7), on Equations (6a) and (6b), we have

$$
\begin{gathered}
-\frac{1}{2} \psi_{r, x_{1} x_{1}}+V_{r} \psi_{r}-V_{\mathrm{i}} \psi_{\mathrm{i}}=E_{r} \psi_{r}-E_{\mathrm{i}} \psi_{\mathrm{i}}, \\
-\frac{1}{2} \psi_{\mathrm{i}, x_{1} x_{1}}+V_{r} \psi_{\mathrm{i}}+V_{\mathrm{i}} \psi_{r}=E_{r} \psi_{\mathrm{i}}+E_{\mathrm{i}} \psi_{r} .
\end{gathered}
$$
[14]

The Ansatz for the wavefunction $\psi(x)$ is assumed as

$$
\psi(x)=\phi(x) \exp [g(x)],
$$

where, $\phi(x)$ and $g(x)$ are written as

$$
\begin{aligned}
& \phi\left(x_{1}, p_{2}\right)=\phi_{r}\left(x_{1}, p_{2}\right)+i \phi_{\mathrm{i}}\left(x_{1}, p_{2}\right), \\
& g\left(x_{1}, p_{2}\right)=g_{r}\left(x_{1}, p_{2}\right)+i g_{\mathrm{i}}\left(x_{1}, p_{2}\right),
\end{aligned}
$$

on substituting Equations (5b), (10a) and (10b) in Equation (9), the real and imaginary parts of the wavefunction are expressed as

$$
\begin{gathered}
\psi_{r}\left(x_{1}, p_{2}\right)=\mathrm{e}^{g_{r}}\left(\phi_{r} \cos g_{\mathrm{i}}-\phi_{\mathrm{i}} \sin g_{\mathrm{i}}\right), \\
\psi_{\mathrm{i}}\left(x_{1}, p_{2}\right)=\mathrm{e}^{g_{r}}\left(\phi_{\mathrm{i}} \cos g_{\mathrm{i}}-\phi_{r} \sin g_{\mathrm{i}}\right) .
\end{gathered}
$$

Then analyticity condition for $g_{r}$ and $g_{\mathrm{i}}$ becomes

$$
g_{r, x_{1}}=g_{\mathrm{i}, p_{2}}, g_{r, p_{2}}=-g_{\mathrm{i}, x_{1}} \text {. }
$$

On implying Equations (11a) and (11b) in Equations (8a) and (8b), one gets

$$
\begin{aligned}
& g_{r, x_{1} x_{1}}+\left(g_{r, x_{1}}\right)^{2}-\left(g_{\mathrm{i}, x_{1}}\right)^{2}+\frac{1}{\left(\phi_{r}^{2}+\phi_{\mathrm{i}}^{2}\right)} \\
& \quad+\phi_{r}\left(\phi_{r, x_{1} x_{1}}+2 \phi_{r, x_{1}} g_{r, x_{1}}-2 \phi_{\mathrm{i}, x_{1}} g_{\mathrm{i}, x_{1}}\right) \\
& \left.\quad+2\left(\phi_{\mathrm{i}, x_{1} x_{1}}+2 \phi_{r, x_{1}} g_{\mathrm{i}, x_{1}}+2 \phi_{\mathrm{i}, x_{1}} g_{r, x_{1}}\right)\right]=0, \\
& g_{\mathrm{i}, x_{1} x_{1}}+2 g_{r, x_{1}} g_{\mathrm{i}, x_{1}}+\frac{1}{\left(\phi_{r}^{2}+\phi_{\mathrm{i}}^{2}\right)} \\
& {\left[\phi_{r}\left(\phi_{\mathrm{i}, x_{1} x_{1}}+2 \phi_{r, x_{1}} g_{\mathrm{i}, x_{1}}+2 \phi_{\mathrm{i}, x_{1}} g_{\mathrm{i}, x_{1}}\right)\right.} \\
& \left.+\phi_{\mathrm{i}}\left(-\phi_{r, x_{1} x_{1}}+2 \phi_{\mathrm{i}, x_{1}} g_{\mathrm{i}, x_{1}}-2 \phi_{r, x_{1}} g_{\mathrm{i}, x_{1}}\right)\right] \\
& +2\left(E_{\mathrm{i}}-V_{\mathrm{i}}\right)=0 .
\end{aligned}
$$

For the ground state solutions, $\phi(x)$ is taken as constant, then Equations (13a) and (13b) reduces to

$$
\begin{gathered}
g_{r, x_{1} x_{1}}+\left(g_{r, x_{1}}\right)^{2}-\left(g_{\mathrm{i}, x_{1}}\right)^{2}+2\left(E_{r}-V_{r}\right)=0, \\
g_{\mathrm{i}, x_{1} X_{1}}+2 g_{r, x_{1}} g_{\mathrm{i}, x_{1}}+2\left(E_{\mathrm{i}}-V_{\mathrm{i}}\right)=0 .
\end{gathered}
$$


By assuming the appropriate ansatz for a given potential, rationalization of Equations (14a) and (14b) provides the ground state solutions, whereas Equations (13a) and (13b) yield the excited state solutions.

\section{Ground State Solutions}

In this section, we are devoted with ground state solutions of a complex octic potential and its variants as

\subsection{Generalized Octic Potential}

Consider a generalized octic potential of the form

$$
\begin{aligned}
V(x)= & a_{0}+a_{1} x+a_{2} x^{2}+a_{3} x^{3} \\
& +a_{4} x^{4}+a_{5} x^{5}+a_{6} x^{6}+a_{7} x^{7}+a_{8} x^{8},
\end{aligned}
$$

where, the coupling parameters $\left(a_{i}, 1 \leq i \leq 8\right)$ are complex constants.

Under the transformation (1), the real and imaginary component of the potential (15) are written as

$$
\begin{aligned}
V_{r}= & a_{0 r}+a_{1 r} x_{1}-a_{1 i} p_{2}+a_{2 r}\left(x_{1}^{2}-p_{2}^{2}\right) \\
& -2 a_{2 i} x_{1} p_{2}+a_{3 r}\left(x_{1}^{3}-3 x_{1} p_{2}^{2}\right)+a_{3 i}\left(p_{2}^{3}-3 x_{1}^{2} p_{2}\right) \\
+ & a_{4 r}\left(x_{1}^{4}-p_{2}^{4}-6 x_{1}^{2} p_{2}^{2}\right)-4 a_{4 i}\left(x_{1} p_{2}^{3}-x_{1}^{3} p_{2}\right) \\
& +a_{5 r}\left(5 x_{1} p_{2}^{4}+x_{1}^{5}-10 x_{1}^{3} p_{2}^{2}\right) \\
& +a_{5 i}\left(-5 x_{1}^{4} p_{2}-p_{2}^{5}+10 x_{1}^{2} p_{2}^{3}\right) \\
+ & a_{6 r}\left(x_{1}^{6}-p_{2}^{6}-15 x_{1}^{2} p_{2}^{4}-15 x_{1}^{4} p_{2}^{2}\right) \\
+ & a_{6 i}\left(-6 x_{1}^{5} p_{2}-6 x_{1} p_{2}^{5}+20 x_{1}^{3} p_{2}^{3}\right) \\
+ & a_{7 r}\left(x_{1}^{7}-7 x_{1} p_{2}^{6}-21 x_{1}^{5} p_{2}^{2}+35 x_{1}^{3} p_{2}^{4}\right) \\
+ & a_{7 i}\left(p_{2}^{7}-7 x_{1}^{6} p_{2}-21 x_{1}^{2} p_{2}^{5}-35 x_{1}^{4} p_{2}^{3}\right) \\
+ & a_{8 r}\left(x_{1}^{8}-p_{2}^{8}-28 x_{1}^{2} p_{2}^{6}-28 x_{1}^{6} p_{2}^{2}\right) \\
+ & a_{8 i}\left(8 x_{1} p_{2}^{7}-8 x_{1}^{7} p_{2}+56 x_{1}^{5} p_{2}^{3}-56 x_{1}^{3} p_{2}^{5}\right), \\
V_{i}= & a_{0 \mathrm{i}}+a_{1 \mathrm{i}} x_{1}-a_{1 r} p_{2}+a_{2 \mathrm{i}}\left(x_{1}^{2}-p_{2}^{2}\right)+2 a_{2 r} x_{1} p_{2} \\
& +a_{3 \mathrm{i}}\left(x_{1}^{3}-3 x_{1} p_{2}^{2}\right)-a_{3 r}\left(p_{2}^{3}-3 x_{1}^{2} p_{2}\right) \\
& +a_{4 \mathrm{i}}\left(x_{1}^{4}-p_{2}^{4}-6 x_{1}^{2} p_{2}^{2}\right)-4 a_{4 r}\left(x_{1} p_{2}^{3}-x_{1}^{3} p_{2}\right) \\
& +a_{5 \mathrm{i}}\left(5 x_{1} p_{2}^{4}+x_{1}^{5}-10 x_{1}^{3} p_{2}^{2}\right) \\
& -a_{5 r}\left(-5 x_{1}^{4} p_{2}-p_{2}^{5}+10 x_{1}^{2} p_{2}^{3}\right) \\
& +a_{6 \mathrm{i}}\left(x_{1}^{6}-p_{2}^{6}-15 x_{1}^{2} p_{2}^{4}-15 x_{1}^{4} p_{2}^{2}\right) \\
& -a_{6 r}\left(-6 x_{1}^{5} p_{2}-6 x_{1} p_{2}^{5}+20 x_{1}^{3} p_{2}^{3}\right) \\
& +a_{7 \mathrm{i}}\left(x_{1}^{7}-7 x_{1} p_{2}^{6}-21 x_{1}^{5} p_{2}^{2}+35 x_{1}^{3} p_{2}^{4}\right) \\
& -a_{7 r}\left(p_{2}^{7}-7 x_{1}^{6} p_{2}-21 x_{1}^{2} p_{2}^{5}-35 x_{1}^{4} p_{2}^{3}\right) \\
& -a_{8 r}\left(x_{1}^{8}-p_{2}^{8}-28 x_{1}^{2} p_{2}^{6}-28 x_{1}^{6} p_{2}^{2}\right) \\
& +a_{8 \mathrm{i}}\left(8 x_{1} p_{2}^{7}-8 x_{1}^{7} p_{2}+56 x_{1}^{5} p_{2}^{3}-56 x_{1}^{3} p_{2}^{5}\right) . \\
&
\end{aligned}
$$

The functional forms of $g_{r}\left(x_{1}, p_{2}\right)$ and $g_{\mathrm{i}}\left(x_{1}, p_{2}\right)$ in consonance with condition (12) are written as

$$
\begin{aligned}
g_{r}= & \frac{1}{2} \alpha_{2}\left(x_{1}^{2}-p_{2}^{2}\right)+\beta_{2} x_{1} p_{2} \\
+ & \frac{1}{3} \alpha_{3}\left(x_{1}^{3}-3 x_{1} p_{2}^{2}\right)-\frac{1}{3} \beta_{3}\left(p_{2}^{3}-3 x_{1}^{2} p_{2}\right) \\
& +\frac{1}{4} \alpha_{4}\left(x_{1}^{4}-p_{2}^{4}-6 x_{1}^{2} p_{2}^{2}\right) \\
- & \beta_{4}\left(x_{1} p_{2}^{3}-x_{1}^{3} p_{2}\right)+\frac{1}{5} \alpha_{5}\left(5 x_{1} p_{2}^{4}+x_{1}^{5}-10 x_{1}^{3} p_{2}^{2}\right) \\
& \quad-\frac{1}{5} \beta_{5}\left(-5 x_{1}^{4} p_{2}-p_{2}^{5}+10 x_{1}^{2} p_{2}^{3}\right), \\
g_{\mathrm{i}}= & \frac{1}{2} \alpha_{2} x_{1} p_{2}+\beta_{2}\left(x_{1}^{2}-p_{2}^{2}\right) \\
- & \frac{1}{3} \beta_{3}\left(x_{1}^{3}-3 x_{1} p_{2}^{2}\right)-\frac{1}{3} \alpha_{3}\left(p_{2}^{3}-3 x_{1}^{2} p_{2}\right) \\
& -\frac{1}{4} \beta_{4}\left(x_{1}^{4}-p_{2}^{4}-6 x_{1}^{2} p_{2}^{2}\right) \\
- & \alpha_{4}\left(x_{1} p_{2}^{3}-x_{1}^{3} p_{2}\right)+\frac{1}{5} \beta_{5}\left(5 x_{1} p_{2}^{4}+x_{1}^{5}-10 x_{1}^{3} p_{2}^{2}\right) \\
& -\frac{1}{5} \alpha_{5}\left(-5 x_{1}^{4} p_{2}-p_{2}^{5}+10 x_{1}^{2} p_{2}^{3}\right),
\end{aligned}
$$

where $\alpha_{i}$ and $\beta_{i}$ are real parameters. Now, substituting Equations (18a) and (18b) in Equations (14a) and (14b), then equating the coefficients of various terms to zero, one gets the following set of 18 non-repeating equations

$$
\begin{aligned}
& E_{r}=a_{0 r}-\frac{1}{2} \alpha_{2}, \\
& E_{i}=a_{0 i}+\frac{1}{2} \beta_{2}, \\
& \alpha_{3}=a_{1 r}, \\
& \beta_{3}=-a_{1 i}, \\
& \alpha_{2}^{2}-\beta_{2}^{2}+3 \alpha_{4}=2 a_{2 r}, \\
& 2 \alpha_{2} \beta_{2}+3 \beta_{4}=-2 a_{2 i}, \\
& \alpha_{2} \alpha_{3}-\beta_{2} \beta_{3}+2 \alpha_{5}=a_{3 r}, \\
& \beta_{2} \alpha_{3}+\alpha_{2} \beta_{3}+2 \beta_{5}=-a_{3 i}, \\
& \alpha_{3}^{2}-\beta_{3}^{2}+2 \alpha_{2} \alpha_{4}-2 \beta_{2} \beta_{4}=2 a_{4 r}, \\
& \alpha_{4} \beta_{2}+\alpha_{2} \beta_{4}+\alpha_{3} \beta_{3}=-a_{4 i}, \\
& \alpha_{3} \alpha_{4}-\beta_{3} \beta_{4}+\alpha_{2} \alpha_{5}-\beta_{2} \beta_{5}=a_{5 r}, \\
& \beta_{2} \alpha_{5}+\alpha_{2} \beta_{5}+\beta_{4} \alpha_{3}+\alpha_{4} \beta_{3}+2 \beta_{5}=a_{5 i}, \\
& \alpha_{4}^{2}-\beta_{4}^{2}+2 \alpha_{3} \alpha_{5}-2 \beta_{3} \beta_{5}=2 a_{6 r}, \\
& \alpha_{3} \beta_{5}+\alpha_{5} \beta_{3}+\alpha_{4} \beta_{4}=-a_{6 i},
\end{aligned}
$$




$$
\begin{array}{r}
\alpha_{4} \alpha_{5}-\beta_{4} \beta_{5}=a_{7 r}, \\
\beta_{4} \alpha_{5}+\alpha_{4} \beta_{5}=-a_{7 i}, \\
\alpha_{5}^{2}-\beta_{5}^{2}=2 a_{8 r}, \\
\alpha_{5} \beta_{5}=-a_{8 i} .
\end{array}
$$

In order to find out the ground state energy eigenvalues and corresponding eigenfunctions, one should solve Equations (19c)-(19r). As there are 16 equations against 8 unknowns (say $\alpha_{i}$ 's and $\beta_{i}$ 's). Therefore in principle one can obtain the solutions of various coefficients $\alpha_{i}$ 's and $\beta_{i}$ 's in terms of the potential parameters $\alpha_{i}$ 's with some constraining relations among $\alpha_{i}$ 's. Then Equations (19q)-(19r) immediately lead to

$$
\alpha_{5}=-a_{8+}, \beta_{5}=a_{8-},
$$

where $a_{8 \pm}=\sqrt{ \pm a_{8 r}+\left|a_{8}\right|}$.

Now Equations (19g)-(19h) and (19m)-(19r) can be immediately solved for the remaining arbitrary constants as

$$
\begin{gathered}
\alpha_{4}=\frac{-1}{2\left|a_{8}\right|}\left(a_{7 r} a_{8+}+a_{7 i} a_{8-}\right), \beta_{4}=\frac{1}{2\left|a_{8}\right|}\left(-a_{7 r} a_{8-}+a_{7 i} a_{8+}\right), \\
\alpha_{3}=\frac{-1}{2\left|a_{8}\right|}\left(h_{r} a_{8+}+h_{i} a_{8-}\right), \beta_{3}=\frac{1}{2\left|a_{8}\right|}\left(-h_{r} a_{8-}+h_{i} a_{8+}\right), \\
\alpha_{2}=\frac{-1}{2|h|^{2}}\left(A_{a h} a_{8+}+D_{a h} a_{8-}-4 K_{a h}\right), \\
\beta_{2}=\frac{1}{2|h|^{2}}\left(D_{a h} a_{8+}-A_{a h} a_{8-}+4 J_{a h}\right),
\end{gathered}
$$

where

$$
\begin{gathered}
h_{r}=a_{6 r}-\frac{1}{4\left|a_{8}\right|^{2}}\left[a_{8 r}\left(a_{7 r}^{2}-a_{7 i}^{2}\right)+2 a_{7 r} a_{7 i} a_{8 i}\right], \\
h_{i}=a_{6 i}+\frac{1}{4\left|a_{8}\right|^{2}}\left[a_{8 i}\left(a_{7 r}^{2}-a_{7 i}^{2}\right)-2 a_{7 r} a_{7 i} a_{8 r}\right], \\
|h|=\sqrt{h_{r}^{2}+h_{i}^{2}}, \\
A_{a h}=a_{3 r} h_{r}+a_{3 i} h_{i}, B_{a h}=a_{3 r} h_{i}+a_{3 i} h_{r}, \\
C_{a h}=a_{3 r} h_{r}-a_{3 i} h_{i}, D_{a h}=a_{3 r} h_{i}-a_{3 i} h_{r}, \\
K_{a h}=a_{8 r} h_{r}+a_{8 i} h_{i}, J_{a h}=a_{8 i} h_{r}-a_{8 r} h_{i},
\end{gathered}
$$

it is to be noted that quantities in Equations (22a)-(22c) are so defined to represent Equations (21a)-(22d) in compact and lucid form. The Equations (19c)-(19f) provides the constraining relations among the potential parameters as

$$
\begin{gathered}
h_{r} a_{8+}+h_{i} a_{8-}=2\left|a_{8}\right| a_{1 r}, \\
h_{r} a_{8-}-h_{i} a_{8+}=2\left|a_{8}\right| a_{1 i}, \\
2\left(A_{a h}^{2}-D_{a h}^{2}\right) a_{8 r}+16\left(K_{a h}^{2}-J_{a h}^{2}\right)+4 A_{a h} D_{a h} a_{8 i} \\
-8\left(A_{a h} K_{a h}+J_{a h} D_{a h}\right) a_{8+} \\
+8\left(A_{a h} J_{a h}-K_{a h} D_{a h}\right) a_{8-}-\frac{3|h|^{4}}{2\left|a_{8}\right|}\left(a_{7 r} a_{8+}+a_{7 i} a_{8-}\right) \\
=2|h|^{4} a_{2 r}, \\
2 A_{a h} D_{a h} a_{8 r}-16 J_{a h} K_{a h}+\left(D_{a h}^{2}-A_{a h}^{2}\right) a_{8 i} \\
+4 J_{a h}\left(A_{a h} a_{8+}+D_{a h} a_{8-}\right) \\
-4 K_{a h}\left(D_{a h} a_{8+}-A_{a h} a_{8-}\right)+\frac{3|h|^{4}}{2\left|a_{8}\right|}\left(a_{7 r} a_{8+}-a_{7 i} a_{8-}\right) \\
=|h|^{4} a_{2 i},
\end{gathered}
$$

in the same way, one can obtain four constraining relations from Equations (19i)-(19l) also. On utilizing the above values of $\alpha_{i j}$ 's and $\beta_{i j}$ 's in (19b) and (19c), the real and imaginary components of the energy eigenvalues for ground state are written as

$$
\begin{aligned}
& E_{r}^{(0)}=a_{0 r}+\frac{1}{2|h|^{2}}\left(A_{a h} a_{8+}+D_{a h} a_{8-}-4 K_{a h}\right), \\
& E_{i}^{(0)}=a_{0 i}+\frac{1}{2|h|^{2}}\left(D_{a h} a_{8+}-A_{a h} a_{8-}+4 J_{a h}\right) .
\end{aligned}
$$

The corresponding eigenfunctions becomes

$$
\begin{aligned}
& \psi^{(0)}(x) \\
& =\exp \left[\frac { - 1 } { 2 | h | ^ { 2 } } \left\{\left(A_{a h}+i D_{a h}\right) a_{8+}+\left(D_{a h}-i A_{a h}\right) a_{8-}\right.\right. \\
& \left.-4 a_{8}\left(h_{r}-i h_{i}\right)\right\} x^{2}-\frac{h}{6\left|a_{8}\right|}\left(a_{8+}-i a_{8+}\right) x^{3} \\
& \left.-\frac{a_{7}}{8\left|a_{8}\right|}\left(a_{8+}-i a_{8+}\right) x^{4}-\frac{1}{5}\left(a_{8+}+i a_{8+}\right) x^{5}\right] .
\end{aligned}
$$

Special case: Under the PT-symmetric condition $\left(x_{1}, p_{2}, p_{1}, x_{2} ; i\right)\left(-x_{1}, p_{2}, p_{1},-x_{2} ;-i\right)$, the potential (15) becomes

$$
\begin{aligned}
V(x)= & a_{0 r}+a_{1 i} x+a_{2 r} x^{2}+a_{3 i} x^{3} \\
& +a_{4 r} x^{4}+a_{5 i} x^{5}+a_{6 r} x^{6}+a_{7 i} x^{7}+a_{8 r} x^{8}
\end{aligned}
$$

In order to extract the information regarding the nature of the energy eigenvalues and eigenfunctions for the potential (27), we follow the same prescription as laid down in general case. Then the energy eigenvalues and eigenfunctions turn out to be 


$$
\begin{aligned}
E_{r(P T)}^{(0)}= & a_{0 r}+\frac{2 \bar{a}_{8 r}\left[a_{3 i} \sqrt{2 \bar{a}_{8 r}}+4 \bar{a}_{8 r}\right]}{\left(4 \bar{a}_{8 r} a_{6 r}-a_{7 i}^{2}\right)}, E_{i(P T)}^{(0)}=0, \\
\psi_{(P T)}^{(0)}(x) & =\exp \left[\frac{2 \bar{a}_{8 r}\left[a_{3 i} \sqrt{2 \bar{a}_{8 r}}+4 \bar{a}_{8 r}\right]}{\left(4 \bar{a}_{8 r} a_{6 r}-a_{7 i}^{2}\right)} x^{2}\right. \\
& \left.-\frac{i\left(4 \bar{a}_{8 r} a_{6 r}-a_{7 i}^{2}\right) x^{3}}{6\left(\bar{a}_{8 r}\right)^{\frac{3}{2}}}+\frac{a_{7 i} x^{4}}{4 \sqrt{2 \bar{a}_{8 r}}}+\frac{i \sqrt{2 \bar{a}_{8 r}}}{5} x^{5}\right]
\end{aligned}
$$

\subsection{Variant of Octic Potential}

Here, we consider the one-dimensional octic potential along with inverse harmonic term as

$$
\begin{aligned}
V(x)= & a_{0}+a_{1} x+a_{2} x^{2}+a_{3} x^{3}+a_{4} x^{4} \\
& +a_{5} x^{5}+a_{6} x^{6}+a_{7} x^{7}+a_{8} x^{8}+\frac{A}{x^{2}},
\end{aligned}
$$

where, the parameters $\left(a_{i}, 1 \leq i \leq 8\right)$ are complex constants.

Again under the transformation (1), the real and imaginary parts of the potential (30) becomes

$$
\begin{gathered}
V_{r}=V_{r_{1}}+A_{r} \frac{\left(x_{1}^{2}-p_{2}^{2}\right)}{\left(x_{1}^{2}+p_{2}^{2}\right)^{2}}+\frac{2 A_{i} x_{1} p_{2}}{\left(x_{1}^{2}+p_{2}^{2}\right)^{2}}, \\
V_{i}=V_{i_{1}}+A_{i} \frac{\left(x_{1}^{2}-p_{2}^{2}\right)}{\left(x_{1}^{2}+p_{2}^{2}\right)^{2}}-\frac{2 A_{r} x_{1} p_{2}}{\left(x_{1}^{2}+p_{2}^{2}\right)^{2}},
\end{gathered}
$$

where, $V_{r_{1}}$ and $V_{\mathrm{i}_{1}}$ are same as given by Equations (16) and (17). The functional form of $g_{r}\left(x_{1}, p_{2}\right)$ and $g_{\mathrm{i}}\left(x_{1}, p_{2}\right)$ in complying with the analyticity condition (12) are written as

$$
\begin{aligned}
& g_{r}=g_{r_{1}}+\gamma \tan ^{-1}\left(\frac{x_{1}}{p_{2}}\right)-\frac{\delta}{2} \log \left(x_{1}^{2}+p_{2}^{2}\right), \\
& g_{\mathrm{i}}=g_{\mathrm{i}_{1}}+\delta \tan ^{-1}\left(\frac{x_{1}}{p_{2}}\right)+\frac{\gamma}{2} \log \left(x_{1}^{2}+p_{2}^{2}\right),
\end{aligned}
$$

where, $g_{r_{1}}$ and $g_{\mathrm{i}_{1}}$ are same as given by Equations (18a) and (18b). After using Equations (31a), (31b), (32a) and (32b) in Equations (14a) and (14b), the rationalization of the final expression yields the following 10 non-repeating equations

$$
\begin{gathered}
E_{r}=a_{0 r}-\frac{1}{2} \alpha_{2}+\alpha_{2} \delta-\beta_{2} \gamma, \\
E_{\mathrm{i}}=a_{0 \mathrm{i}}+\frac{1}{2} \beta_{2}-\alpha_{2} \gamma-\beta_{2} \delta, \\
\alpha_{3}-\alpha_{3} \delta+\beta_{3} \gamma=a_{1 r}, \\
\beta_{3}-\alpha_{3} \gamma-\beta_{3} \delta=-a_{1 \mathrm{i}}, \\
\alpha_{2}^{2}-\beta_{2}^{2}+3 \alpha_{4}-2 \alpha_{4} \delta+2 \beta_{4} \gamma=2 a_{2 r},
\end{gathered}
$$

$$
\begin{gathered}
2 \alpha_{2} \beta_{2}+3 \beta_{4}-2 \alpha_{4} \gamma-2 \beta_{4} \delta=-2 a_{2 \mathrm{i}}, \\
\alpha_{2} \alpha_{3}-\beta_{2} \beta_{3}+2 \alpha_{5}-\alpha_{5} \delta+\beta_{5} \gamma=a_{3 r}, \\
\beta_{2} \alpha_{3}+\alpha_{2} \beta_{3}+2 \beta_{5}-\alpha_{5} \gamma-\beta_{5} \delta=-a_{3 \mathrm{i}} \\
\delta^{2}-\gamma^{2}+\delta=2 A_{r}, \\
\gamma-2 \gamma \delta=-A_{1} .
\end{gathered}
$$

Now, Equations (33g) and (33h) lead to

$$
\alpha_{2}=\frac{-1}{2|h|^{2}}\left[A_{a h} a_{8+}+D_{a h} a_{8-}+4\left\{\left(A_{r}-1\right) K_{a h}+A_{r} J_{a h}\right\}\right] \text {, }
$$

$$
\beta_{2}=\frac{1}{2|h|^{2}}\left[D_{a h} a_{8+}-A_{a h} a_{8-}+4\left\{\left(A_{r}-1\right) J_{a h}-A_{r} K_{a h}\right\}\right],
$$

where, Equations (33i) and (33j) can be solved for $\gamma$ and $\delta$. For convenience, we choose $\gamma=\delta$, then Equations (33j) yields $\gamma=\delta=2 A_{r}$.

The constraining relations can be derived from Equations (33c)-(33f). Finally employing the values of various ansatz parameters in Equations (33a) and (33b), the real and imaginary part of energy eigenvalues in the ground state are written as

$$
\begin{aligned}
E_{r}^{(0)}= & a_{0 r} \\
& +\frac{1}{2|h|^{2}}\left[A_{a h} a_{8+}+D_{a h} a_{8-}+4\left\{\left(A_{r}-1\right) K_{a h}+A_{r} J_{a h}\right\}\right. \\
& -\frac{2 A_{r}}{|h|^{2}}\left\{\left(A_{a h}-D_{a h}\right) a_{8+}+\left(A_{a h}-D_{a h}\right) a_{8-}\right\} \\
& \left.+4\left\{\left(A_{r}-1\right)\left(J_{a h}+K_{a h}\right)+4 A_{r}\left(J_{a h}-K_{a h}\right)\right\}\right],
\end{aligned}
$$

$$
\begin{aligned}
E_{\mathrm{i}}^{(0)}= & a_{0 \mathrm{i}}+\frac{1}{2|h|^{2}}\left[A_{a h} a_{8-}-D_{a h} a_{8+}+4\left\{\left(A_{r}-1\right) J_{a h}\right.\right. \\
& \left.+A_{r} K_{a h}\right\}-\frac{2 A_{r}}{|h|^{2}}\left\{-\left(A_{a h}-D_{a h}\right) a_{8+}+\left(A_{a h}-D_{a h}\right) a_{8-}\right\} \\
& \left.+4\left\{\left(A_{r}-1\right)\left(J_{a h}-K_{a h}\right)-4 A_{r}\left(J_{a h}+K_{a h}\right)\right\}\right],
\end{aligned}
$$

The corresponding eigenfunctions becomes

$$
\begin{aligned}
& \psi^{(0)}(x)=\left(x_{1}^{2}+p_{2}^{2}\right)^{A_{r}(i-1)} \exp \left[\frac{-1}{2|h|^{2}}\left(A_{a h}-i D_{a h}\right) a_{8+}\right. \\
& +\left(D_{a h}+i A_{a h}\right) a_{8-}+4\left(A_{r}-1\right)\left(K_{a h}+i J_{a h}\right) \\
& -4 A_{r}\left(J_{a h}-i K_{a h}\right) x^{2}-\frac{h}{6\left|a_{8}\right|}\left(a_{8+}-i a_{8+}\right) x^{3}-\frac{a_{7}}{8\left|a_{8}\right|} \\
& \left.\times\left(a_{8+}-i a_{8+}\right) x^{4}-\frac{1}{5}\left(a_{8+}+i a_{8+}\right) x^{5}+2 A_{r} \tan ^{-1}\left(\frac{x_{1}}{p_{2}}\right)\right],
\end{aligned}
$$

After using the condition 
$\left(x_{1}, p_{2}, p_{1}, x_{2} ; i\right)\left(-x_{1}, p_{2}, p_{1},-x_{2} ;-i\right)$, the PT-symmetric solutions for the potential (30) are given by

$$
\begin{aligned}
& E_{r(P T)}^{(0)}= a_{0 r} \\
&+\frac{-2\left(-2+\sqrt{1+8 A_{r}}\right) \bar{a}_{8 r}\left[a_{3 i} \sqrt{2 \bar{a}_{8 r}}-\left(-5+\sqrt{1+8 A_{r}}\right) \bar{a}_{8 r}\right]}{\left(4 \bar{a}_{8 r} a_{6 r}-a_{7 i}^{2}\right)} \\
& E_{i(P T)}^{(0)}=0, \\
& \\
& \psi_{(P T)}^{(0)}(x)=\left(x_{1}^{2}+p_{2}^{2}\right)^{-\frac{\delta}{2}} \\
& \quad \times \exp \left[\frac{-2 \bar{a}_{8 r}\left[a_{3 i} \sqrt{2 \bar{a}_{8 r}}-\left(-5+\sqrt{1+8 A_{r}}\right) \bar{a}_{8 r}\right]}{\left(4 \bar{a}_{8 r} a_{6 r}-a_{7 i}^{2}\right)} x^{2}\right. \\
& \quad-\frac{i\left(4 \bar{a}_{8 r} a_{6 r}-a_{7 i}^{2}\right) x^{3}}{6\left(\bar{a}_{8 r}\right)^{\frac{3}{2}}}+\frac{a_{7 i}}{4 \sqrt{2 \bar{a}_{8 r}}} x^{4} \\
&\left.+\frac{i \sqrt{2 \bar{a}_{8 r}}}{5} x^{5}+i \tan ^{-1}\left(x_{1} / p_{2}\right)\right] .
\end{aligned}
$$

\section{Excited State Solutions}

To compute the eigenvalues and eigenfunctions for the first excited state, we follow the same prescription as adopted in earlier section. The functional form of $\phi(x)$ for the first excited state is taken as

$$
\phi(x)=\alpha x+\eta,
$$

where, $\alpha$ and $\eta$ are considered as real constants. Then under the transformation (1), Equation (40) reduces to

$$
\phi_{r}\left(x_{1}, p_{2}\right)=\alpha x_{1}+\eta, \phi_{\mathrm{i}}\left(x_{1}, p_{2}\right)=\alpha p_{2} .
$$

\subsection{Generalized Octic Potential}

In order to compute the energy eigenvalues and associated eigenfunctions for the first excited state of the potential (15), we use the same functional forms of $g_{r}$ and $g_{\mathrm{i}}$ as mentioned in Equations (18a) and (18b). Then inserting Equations (18a), (18b) and (41) in Equations (13a) and (13b), the rationalization of the resultant expression yields the following non-repeating equations in addition to Equations (19c), (19d) and (19i)-(19r) as

$$
\begin{gathered}
E_{r}^{(1)}=a_{0 r}-\frac{3}{2} \alpha_{2}, \\
E_{i}^{(1)}=a_{0 i}+\frac{3}{2} \beta_{2}, \\
\alpha_{2}^{2}-\beta_{2}^{2}+5 \alpha_{4}=2 a_{2 r},
\end{gathered}
$$

$$
\begin{array}{r}
2 \alpha_{2} \beta_{2}+5 \beta_{4}=-2 a_{2 i}, \\
\alpha_{2} \alpha_{3}-\beta_{2} \beta_{3}+3 \alpha_{5}=a_{3 r} \\
\beta_{2} \alpha_{3}+\alpha_{2} \beta_{3}+3 \beta_{5}=-a_{3 i} .
\end{array}
$$

The Equations (42c) and (42d) gives the constraining relations as

$$
\begin{aligned}
& 2\left(A_{a h}^{2}-D_{a h}^{2}\right) a_{8 r}+16\left(K_{a h}^{2}-J_{a h}^{2}\right) \\
& +4 A_{a h} D_{a h} a_{8 \mathrm{i}}-8\left(A_{a h} K_{a h}+J_{a h} D_{a h}\right) a_{8+} \\
& +8\left(A_{a h} J_{a h}-K_{a h} D_{a h}\right) a_{8-}-\frac{5|h|^{4}}{2\left|a_{8}\right|}\left(a_{7 r} a_{8+}+a_{7 \mathrm{i}} a_{8-}\right) \\
& =2|h|^{4} a_{2 r}, \\
& 2 A_{a h} D_{a h} a_{8 r}-16 J_{a h} K_{a h}+\left(D_{a h}^{2}-A_{a h}^{2}\right) a_{8 \mathrm{i}} \\
& +4 J_{a h}\left(A_{a h} a_{8+}+D_{a h} a_{8-}\right) \\
& -4 K_{a h}\left(D_{a h} a_{8+}-A_{a h} a_{8-}\right)+\frac{5|h|^{4}}{2\left|a_{8}\right|}\left(a_{7 r} a_{8+}-a_{7 \mathrm{i}} a_{8-}\right) \\
& =|h|^{4} a_{2 \mathrm{i}}
\end{aligned}
$$

whereas, Equations (42e) and (42f) can be solved for $\alpha_{2}$ and $\beta_{2}$ as

$$
\begin{gathered}
\alpha_{2}=\frac{-1}{|h|^{2}}\left(A_{a h} a_{8+}+D_{a h} a_{8-}-6 K_{a h}\right), \\
\beta_{2}=\frac{-1}{|h|^{2}}\left(D_{a h} a_{8+}-A_{a h} a_{8-}+6 J_{a h}\right) .
\end{gathered}
$$

After inserting the values of various Ansatz parameters in Equations (42a) and (42b), the energy eigenvalues for the first excited state are

$$
\begin{gathered}
E_{r}^{(1)}=a_{0 r}+\frac{3}{2|h|^{2}}\left(A_{a h} a_{8+}+D_{a h} a_{8-}-6 K_{a h}\right), \\
E_{\mathrm{i}}^{(1)}=a_{0 \mathrm{i}}+\frac{3}{2|h|^{2}}\left(D_{a h} a_{8+}-A_{a h} a_{8-}+6 J_{a h}\right),
\end{gathered}
$$

and the corresponding eigenfunctions becomes

$$
\begin{aligned}
& \psi^{(1)}(x) \\
& =\exp \left[\frac { - 1 } { 2 | h | ^ { 2 } } \left\{\left(A_{a h}+i D_{a h}\right) a_{8+}+\left(D_{a h}-i A_{a h}\right) a_{8-}\right.\right. \\
& \left.-6 a_{8}\left(h_{r}-i h_{i}\right)\right\} x^{2}-\frac{h}{6\left|a_{8}\right|}\left(a_{8+}-i a_{8+}\right) x^{3} \\
& \left.-\frac{a_{7}}{8\left|a_{8}\right|}\left(a_{8+}-i a_{8+}\right) x^{4}-\frac{1}{5}\left(a_{8+}+i a_{8+}\right) x^{5}\right]
\end{aligned}
$$

Special case: The PT-symmetric solutions for the first excited state are given by 


$$
\begin{aligned}
E_{r(P T)}^{(1)}= & a_{0 r}+\frac{6 \bar{a}_{8 r}\left[a_{3 i} \sqrt{2 \bar{a}_{8 r}}+6 \bar{a}_{8 r}\right]}{\left(4 \bar{a}_{8 r} a_{6 r}-a_{7 i}^{2}\right)}, E_{i(P T)}^{(1)}=0(47 \mathrm{a}) \\
\psi_{(P T)}^{(1)}(x)= & \exp \left[\frac{2 \bar{a}_{8 r}\left[a_{3 i} \sqrt{2 \bar{a}_{8 r}}+6 \bar{a}_{8 r}\right]}{\left(4 \bar{a}_{8 r} a_{6 r}-a_{7 i}^{2}\right)} x^{2}\right. \\
& \left.-\frac{i\left(4 \bar{a}_{8 r} a_{6 r}-a_{7 i}^{2}\right) x^{3}}{6\left(\bar{a}_{8 r}\right)^{\frac{3}{2}}}+\frac{a_{7 i} x^{4}}{4 \sqrt{2 \bar{a}_{8 r}}}+\frac{i \sqrt{2 \bar{a}_{8 r}}}{5} x^{5}\right]
\end{aligned}
$$

\subsection{Variant of Octic Potential}

To compute the energy eigenvalues and associated eigenfunctions for the first excited state of the potential (30), we use the same polynomial forms of $g_{r}$ and $g_{\text {i }}$ as in Equations (32a) and (32b). Then implying these equations in $g_{r}$ and $g_{\mathrm{i}}$ in Equations (13a) and (13b), the rationalization of the resultant expression yields the following equations in addition to Equations (19c), (19d) and (19i)-(19r) as

$$
\begin{gathered}
E_{r}=a_{0 r}-\frac{3}{2} \alpha_{2}+\alpha_{2} \delta-\beta_{2} \gamma, \\
E_{\mathrm{i}}=a_{0 \mathrm{i}}+\frac{3}{2} \beta_{2}-\alpha_{2} \gamma-\beta_{2} \delta, \\
2 \alpha_{3}-\alpha_{3} \delta+\beta_{3} \gamma=a_{1 r}, \\
2 \beta_{3}-\alpha_{3} \gamma-\beta_{3} \delta=-a_{1 \mathrm{i}}, \\
\alpha_{2}^{2}-\beta_{2}^{2}+5 \alpha_{4}-2 \alpha_{4} \delta+2 \beta_{4} \gamma=2 a_{2 r}, \\
2 \alpha_{2} \beta_{2}+5 \beta_{4}-2 \alpha_{4} \gamma-2 \beta_{4} \delta=-2 a_{2 \mathrm{i}}, \\
\alpha_{2} \alpha_{3}-\beta_{2} \beta_{3}+3 \alpha_{5}-\alpha_{5} \delta+\beta_{5} \gamma=a_{3 r}, \\
\beta_{2} \alpha_{3}+\alpha_{2} \beta_{3}+3 \beta_{5}-\alpha_{5} \gamma-\beta_{5} \delta=-a_{3 \mathrm{i}}, \\
\delta^{2}-\gamma^{2}-\delta=2 A_{r}, \\
\gamma-2 \gamma \delta=-A_{1} .
\end{gathered}
$$

Now, Equations (48g) and (48h) yield

$$
\begin{array}{r}
\alpha_{2}=\frac{-1}{|h|^{2}}\left[A_{a h} a_{8+}+D_{a h} a_{8-}+2\left(3+2 A_{r}\right) K_{a h}+4 A_{r} J_{a h}\right], \\
\beta_{2}=\frac{1}{2|h|^{2}}\left[-D_{a h} a_{8+}+A_{a h} a_{8-}+2\left(3+2 A_{r}\right) J_{a h}-4 A_{r} K_{a h}\right],
\end{array}
$$

where, the Equations (48i) and (48j) can be solved for $\gamma$ and $\delta$. For convenience, we set $\gamma=\delta$, then Equation (48j) yields $\gamma=\delta=-2 A_{r}$.

Finally, substituting the values of various ansatz parameters in Equations (48a) and (48b), the real and imaginary part of energy eigenvalues for the ground state are written as

$$
\begin{aligned}
E_{r}^{(1)}= & a_{0 r}+\frac{3}{2|h|^{2}}\left[A_{a h} a_{8+}+D_{a h} a_{8-}+2\left(3+2 A_{r}\right) K_{a h}\right. \\
& +4 A_{r} J_{a h}+\frac{2 A_{r}}{|h|^{2}}\left\{\left(A_{a h}+D_{a h}\right) a_{8+}+\left(A_{a h}+D_{a h}\right) a_{8-}\right\} \\
& \left.-2\left(3+2 A_{r}\right)\left(J_{a h}+K_{a h}\right)-4 A_{r}\left(J_{a h}-K_{a h}\right)\right], \\
E_{i}^{(1)}= & a_{0 i}+\frac{3}{2|h|^{2}}\left[-D_{a h} a_{8+}+A_{a h} a_{8-}+2\left(3+2 A_{r}\right) J_{a h}\right. \\
& -4 A_{r} K_{a h}-\frac{2 A_{r}}{|h|^{2}}\left\{\left(A_{a h}-D_{a h}\right) a_{8+}+\left(-A_{a h}+D_{a h}\right) a_{8-}\right\} \\
& \left.-2\left(3+2 A_{r}\right)\left(-J_{a h}+K_{a h}\right)+4 A_{r}\left(J_{a h}+K_{a h}\right)\right],
\end{aligned}
$$

The corresponding eigenfunctions becomes

$$
\begin{aligned}
& \psi^{(1)}(x)=\left(x_{1}^{2}+p_{2}^{2}\right)^{A_{r}(1-i)} \\
& \times \exp \left[\frac { - 1 } { 2 | h | ^ { 2 } } \left\{\left(A_{a h}-i D_{a h}\right) a_{8+}+\left(D_{a h}+i A_{a h}\right) a_{8-}\right.\right. \\
& \left.+2\left(3+2 A_{r}\right)\left(K_{a h}+i J_{a h}\right)+4 A_{r}\left(J_{a h}-i K_{a h}\right)\right\} x^{2} \\
& -\frac{h}{6\left|a_{8}\right|}\left(a_{8+}-i a_{8+}\right) x^{3}-\frac{a_{7}}{8\left|a_{8}\right|}\left(a_{8+}-i a_{8+}\right) x^{4} \\
& \left.-\frac{1}{5}\left(a_{8+}+i a_{8+}\right) x^{5}+2 A_{r}(1+i) \tan ^{-1}\left(x_{1} / p_{2}\right)\right] .
\end{aligned}
$$

The $P T$-symmetric excited state solutions for the potential (30) turn out to be

$$
\begin{aligned}
& E_{r(P T)}^{(1)}=a_{0 r} \\
& +\frac{\left(-2+\sqrt{1+8 A_{r}}\right) \bar{a}_{8 r}\left[a_{3 i} \sqrt{2 \bar{a}_{8 r}}+\left(5-\sqrt{1+8 A_{r}}\right) \bar{a}_{8 r}\right]}{\left(4 \bar{a}_{8 r} a_{6 r}-a_{7 i}^{2}\right)}, \\
& E_{i(P T)}^{(1)}=0
\end{aligned}
$$

$\psi_{(P T)}^{(1)}(x)=\left(x_{1}^{2}+p_{2}^{2}\right)^{-\frac{\delta}{2}}$

$\times \exp \left[\frac{-2 \bar{a}_{8 r}\left[a_{3 i} \sqrt{2 \bar{a}_{8 r}}-\left(-5+\sqrt{1+8 A_{r}}\right) \bar{a}_{8 r}\right]}{\left(4 \bar{a}_{8 r} a_{6 r}-a_{7 i}^{2}\right)} x^{2}\right.$

$-\frac{i\left(4 \bar{a}_{8 r} a_{6 r}-a_{7 i}^{2}\right) x^{3}}{6\left(\bar{a}_{8 r}\right)^{\frac{3}{2}}}$

$\left.-\frac{a_{7 i}}{4 \sqrt{2 \bar{a}_{8 r}}} x^{4}-\frac{i \sqrt{2 \bar{a}_{8 r}}}{5} x^{5}+i \tan ^{-1}\left(x_{1} / p_{2}\right)\right]$ 


\section{Conclusion}

In the present work, we have computed the quasi-exact solutions of the SE for one-dimensional octic potential and its variants by using ECPS approach. The non-hermiticity arising is not only due to the potential parameters but the underlying phase space is also considered as complex. The PT-symmetry discussed here is of generalized nature which in certain limit (for real $x$ and $p$ ) reduces to conventional $P T$-symmetry, besides the complexity of the phase space produced by (1), the complexity of the potential parameters is also taken into account. It is also emphasized that solutions of the ASE in the above mentioned cases are obtained only in the presence of certain constraining relation among the potential parameters, such constraining relations give rise to bound state energies of a system. It is found that the imaginary part of the energy eigenvalues always vanishes for the solvable cases of ASE as long as all the potential parameters are real [14-16]. However, for the PT-symmetric potentials, the energy eigenvalues are found real, even if the concerned potential possesses complex couplings parameters. The result obtained in this manner coincides with those computed from the invariant of Hamiltonian under $P T$ operation. The interesting aspect of this method is an account of the complex coupling parameters in the potential in addition to the complex phase space, such feature of the method lead to complex spectra. If $P T$-symmetric version of the concerned potential is taken into account, then it is found that imaginary part of the complex eigenvalue vanishes after imposing certain restrictions on the complex coupling parameters of the potential. It is observed that real and imaginary parts of the eigenvalues follow just opposite ordering for the discrete energy level by retaining the conventional ordering for the magnitude of the eigenvalues.

\section{Acknowledgements}

The author expresses his gratitude to Prof. S. C. Mishra and Dr. Fakir Chand, Department of Physics, Kurukshetra University, Kurukshetra (India) for their valuable suggestions regarding the manuscript. He is also thankful to the referees for several useful comments which helped to considerable improvement and fine tuning of some of the ideas in the original version of the paper.

\section{REFERENCES}

[1] T. J. Hollowood, "Solitons in Affine Toda Theories," Nuclear Physics B, Vol. 384, No. 3, 1992, pp. 523-540. doi:10.1016/0550-3213(92)90579-Z
[2] D. R. Nelson and N. M. Shnerb, "Non-Hermitian Localization and Population Biology,” Physical Review E, Vol. 58, No. 2, 1998, pp. 1383-1403.

[3] N. Hatano and D. R. Nelson, "Localization Transitions in Non-Hermitian Quantum Mechanics,” Physical Review Letters, Vol. 77, No. 3, 1996, pp. 570-573.

[4] N. Hatano and D. R. Nelson, "Vortex Pinning and NonHermitian Quantum Mechanics,” Physical Review B, Vol. 56, No. 14, 1997, pp. 8651-8673.

[5] H. Feshbach, C. E. Porter and V. F. Weisskopf, "Model for Nuclear Reactions with Neutrons," Physical Review Letters, Vol. 96, No. 2, 1954, pp. 448-464.

[6] F. Verheest, "Nonlinear Wave Interaction in a Complex Hamiltonian Formalism,” Journal of Physics A: Mathematical and General, Vol. 20, No. 1, 1987, pp. 103-110. doi:10.1088/0305-4470/20/1/019

[7] N. N. Rao, B. Buti and S. B. Khadkikar, "Hamiltonian Systems with Indefinite Kinetic Energy,” PramanaJournal of Physics, Vol. 27, No. 4, 1986, pp. 497-505. doi:10.1007/BF02846877

[8] R. S. Kaushal and H. J. Korsch, "Some Remarks on Complex Hamiltonian Systems,” Physics Letters A, Vol. 276, No. 1-4, 2000, pp. 47-51.

[9] R. S. Kaushal and S. Singh, "Construction of Complex Invariants for Classical Dynamical Systems," Annals of Physics (New York), Vol. 288, No. 2, 2001, pp. 253-276.

[10] C. M. Bender and A. Turbiner, "Analytic Continuation of Eigenvalue Problems,” Physics Letters A, Vol. 173, No. 6, 1993, pp. 442-446. doi:10.1016/0375-9601(93)90153-Q

[11] C. M. Bender and S. Boettcher, "Real Spectra in NonHermitian Hamiltonians Having PT-Symmetry," Physical Review Letters, Vol. 80, No. 24, 1998, pp. 5243-5246.

[12] A. L. Xavier Jr. and M. A. M. de Aguiar, "Complex Trajectories in the Quartic Oscillator and Its Semiclassical Coherent-State,” Annals of Physics (New York), Vol. 252, No. 2, 1996, pp. 458-476. doi:10.1006/aphy.1996.0141

[13] A. L. Xavier Jr. and M. A. M. de Aguiar, "Phase Space Approach to the Tunnel Effect: A New Semiclassical Traversal Time,” Physical Review Letters, Vol. 79, No. 18, 1997, pp. 3323-3326.

[14] R. S. Kaushal, "On the Quantum Mechanics of Complex Hamiltonian Systems in One Dimension," Journal of Physics A: Mathematical and General, Vol. 34, No. 49, 2001, pp. L709-L714. doi:10.1088/0305-4470/34/49/104

[15] R. S. Kaushal and Parthasarthi, "Quantum Mechanics of Complex Hamiltonian Systems in One Dimension,” Journal of Physics A: Mathematical and General, Vol. 35, No. 41, 2002, pp. 8743-8761. doi:10.1088/0305-4470/35/41/308

[16] Parthasarthi and R. S. Kaushal, "Quantum Mechanics of Complex Sextic Potential in One Dimension,” Physica Scripta, Vol. 68, No. 2, 2003, pp. 115-127. 